of the topics one could do better by turning to more specialised sources. As a book of its kind, however, it is one of the best.

It is clear from the evidence presented that Cybernetics still awaits its breakthroughs. As a body of theory it has little to offer and seems to have taken almost everything it has from other disciplines. The developments envisaged by Wiener, Ashby, and others, that can result from the interaction of ideas from biology and technology do, of course, continue to take

\section{Heterocyclic chemistry}

Stereochemistry of Heterocyclic Compounds. Part 2: Oxygen, Sulfur, Mixed $\mathrm{N}, \mathrm{O}$, and $\mathrm{S}$, and Phosphorus Heterocycles. By W. L. F. Armarego. Pp, xviii +494 . (Wiley-Interscience: London and New York, 1977.) $£ 32.65 ; \$ 54$.

Although the literature of organic chemistry includes many comprehensive surveys of the stereochemistry and conformational analysis of carbocyclic compounds, scant attention has hitherto been given to the corresponding aspects of heterocyclic chemistry. Numerous discussions of isolated topics in this vast, comparatively neglected area are scattered throughout the review literature, but the present two-volume set appears to be the first monograph to attempt a review of this field as a whole. This second volume is devoted to the stereochemistry of oxygen, sulphur, mixed nitrogen, oxygen and sulphur heterocycles, and a final chapter deals with phosphorus heterocycles.

The organisation of the volume is the same as that of the first, even to the extent that the first brief, introductory chapter, which explains the order of discussion of the data, is identical in the two volumes. Each section begins with a discussion of the stereochemical aspects of the synthesis of compounds in the group, then follows a discussion of the configuration, and then the conformation, of the compounds, and finally stereochemical aspects of their reactions are discussed. The whole project is one of daunting proportions; this is amply illustrated by the fact that this second volume alone contains a bibliography of over 2,400 references, which cover the subject completely up to the end of 1974 , and incompletely for 1975. Presumably because of the enormous volume of material to be reviewed, the final chapter has been contributed by M. J. Gallagher.

The coverage of this material in under 450 pages of text has resulted in inevitable restrictions; the style is of place; but, perhaps not, as they had hoped, uncer the heading of Cybernetics. Ironically, there is the consolation that the "arbitrary divisions" which, some thirty years ago, marked out this new field of science are not likely to become hard-set, and thereby to obstruct future changes in the internal boundaries of science.

\section{Zakian}

V. Zakian is Lecturer at the University of Manchester Institute of Science and Technology, Manchester, UK.

necessity compressed, but it is clear, critical, and very readable. Where earlier authoritative reviews exist, the author has been concerned primarily with updating them. Important stereochemical principles and illustrations of syntheses and reactions are taken mainly from the behaviour of model compounds, and a discussion of the vast number of natural products is not attempted. In the present context this includes, for example, furanose and pyranose carbohydrates, penicillins and cephalosporins. Steroidal epoxides are also among the groups omitted; however, in all these areas, the excellent bibliography provides a ready entry into these aspects of the subject, as well as providing a comprehensive bibliography for readers who wish to pursue the subject matter of the text in greater depth.

The book is beautifully produced, and typographical errors are rare. The formulae have been very skilfully drawn by the author's talented amanuensis, the heteroatoms and substituent groups being inserted manually rather than in the usual type-face. This has presumably resulted in some economy in production, and it has also obviated one possible source of errors, without any sacrifice of production standards. There is a moderately detailed subject index, which also includes the names of authors mentioned in the text; there is no separate, complete, author index.

This volume certainly fills a very important gap in the review literature of heterocyclic chemistry, and since, with its companion volume on nitrogen heterocycles, it heralds the introduction of a new series of monographs on general heterocyclic chemistry, each volume in which will survey one aspect of the whole field of heterocyclic chemistry, it is to be particularly welcomed. It will be immensely useful to advanced undergraduates and to research workers in heterocyclic chemistry, and it surely belongs in every chemistry library and heterocyclic research laboratory.

J. E. Saxton

J.E. Saxton is Senior Lecturer in Organic Chemistry at the University of Leeds, UK.
Biological tools

Introduction to the Spectroscopy of Biological Polymers. Edited by D. W. Jones. Pp. xii +328 . (Academic: London and New York, 1977.) £11.60; $\$ 25.50$.

Specrroscopic techniques have played no less an important part in the structural studies of biological macromolecules than they have in more classical studies of simpler molecules, and the range of spectroscopies, if differing in emphasis, has been equally as wide. Consequently, an author or editor aiming to produce a book that covers all or most of the spectroscopic techniques used in studying biological polymers sets himself an unenviable task. $\mathrm{He}$ not only attempts a synthesis of several highly specialised subjects but does so, unlike his many predecessors who have undertaken similar enterprises in a purely chemical context, knowing he can make very few assumptions about his readers' knowledge of mathematics and physics.

The editor of this work has overcome the problem of the multidisciplinary nature of his subject by choosing an author eminent in each field to contribute a chapter on that speciality. $\mathrm{He}$ has sandwiched these chapters between a general introduction to molecular spectroscopy and a concluding chapter briefly describing some examples that illustrate the power of combining several spectroscopic techniques to tackle a specific biological problem. The approach throughout is descriptive and not mathematical, and several difficult physical concepts are explained clearly.

The most original aspect of the book is the strong emphasis placed on the use of vibrational spectroscopy. The chapter on Raman spectroscopy is particularly welcome as advances in laser technology over the past decade have madie this technique a powerful biological tool. No less welcome and more suprising are the two chapters on infrared spectroscopy. They illustrate the potential of this technique especially when combined with Fouriertransform analysis. They also link the work done on biological polymers with that done on the industrially important synthetic polymers. The remaining chapters cover absorption, emission, optical rotation, circular dichroism, nuclear magnetic resonance, electron spin reasonance and Mössbauer spectroscopy. Michael T. Flanagan

$M . T$. Flanagan, until recently a member of the Biophysics Division of the National Institute of Medical Research, London, is now with Standard Telecommunication Laboratories, Harlow, UK. 